\title{
Key Industries Ceramics and Glass
}

For the ceramics and glass industry, a series of topics will come to the fore this autumn, for example because of the upcoming trade fairs and conferences: In September the Tecnargilla takes place in Rimini, Italy, at the same time as the $61^{\text {st }}$ International Refractories Colloquium in Aachen, Germany - and then in October glasstec in Düsseldorf, Germany. A number of interesting conferences and trade fairs outside Europe are also organised. Some of them are listed in the front part of the magazine.

However, not only ceramics are the focus of this issue, but also glass like ceramics a non-metallic inorganic material, but with an amorphous structure. While containers made of conventional ceramics (and porcelain) have been used as storage media for thousands of years, technical ceramics are increasingly being used for modern packaging solutions. Similarly, glass has been known for centuries as a suitable and decorative storage medium. Glass containers are inert: taste and freshness are retained, foreign substances are not released into the contents. Today glass is still a popular material in the field of filling technology for pharmaceutical and food products. At the same time, the material scores points in comparison to plastics with its recycling properties. But glass and ceramics can do much more.

The ceramics and glass industry is also one of the energy-intensive industries and must therefore deal with issues such as $\mathrm{CO}_{2}$ emissions and energy reduction. An important task - and challenge - for the industry is "sustainable production with a low carbon footprint" in the coming years.

Energy-intensive industries are considered key industries and job engines. As suppliers of basic materials they are positioned at the beginning of a value chain from which highly innovative products can be made. At the end - in turn these products can contribute to the great challenges facing our society: new forms of urbanisation and mobility, communication and digitisation, energy management and sustainability.

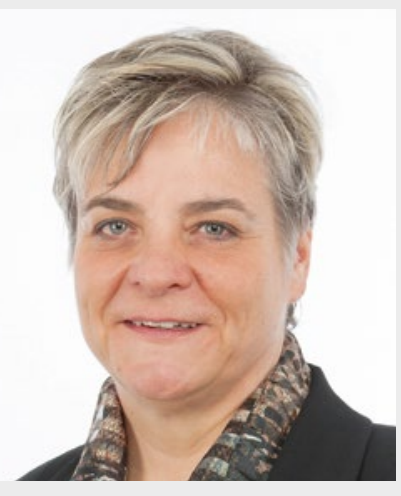

Dr. Wiebke Sanders Responsible Editor wiebke.sanders@springernature.com 